\title{
Bragg gratings in normal and reduced diameter high birefringence fibre optics
}

\author{
Ilda Abe ${ }^{1}$, Orlando Frazão ${ }^{2}$, Marcelo W Schiller ${ }^{1}$, \\ Rogério N Nogueira $^{3}$, Hypolito J Kalinowski ${ }^{4}$ and João L Pinto ${ }^{1,3}$ \\ ${ }^{1}$ Departamento de Física, Universidade de Aveiro, Campus Universitário de Santiago, \\ 3810-193 Aveiro, Portugal \\ ${ }^{2}$ Unidade de Optoelectrónica e Sistemas Electrónicos, INESC Porto, Rua Campo Alegre, \\ 687 4169-007 Porto, Portugal \\ ${ }^{3}$ Instituto de Telecomunicações, Campus Universitário de Santiago, 3810-193 Aveiro, \\ Portugal \\ ${ }^{4}$ Universidade Tecnológica Federal do Paraná, Av. Sete de Setembro, \\ 3165 80230-901 Curitiba, Brazil \\ E-mail: ilda@fis.ua.pt
}

Received 23 January 2006, in final form 16 February 2006

Published 8 May 2006

Online at stacks.iop.org/MST/17/1477

\begin{abstract}
Fibre optic Bragg gratings (FBGs) written in normal and reduced diameter high birefringence (HiBi) fibres are studied. Chemical etching is used to reduce the diameter of fibres while the optical properties of the FBG spectrum are measured. The results obtained agree qualitatively with the stress enhanced chemical etching. The birefringence of the fibre is determined as a function of the diameter. Optical characterization of the FBG under transverse strain and temperature is also performed. The results obtained show the feasibility of the simultaneous measurement of those parameters with a HiBi FBG sensor.
\end{abstract}

Keywords: Bragg gratings, high birefringence, fibre optics

\section{Introduction}

Sensors capable of measuring simultaneously several physical parameters have increased importance in today's technological world. In particular, there are various applications of such sensors in civil, mechanical, biomedical or aeronautical engineering, where measurement of both strain and temperature is required [1]. The fibre optic Bragg grating (FBG) $[2,3]$ has widespread application in sensors for those parameters, as the Bragg wavelength (and the grating reflection spectrum) depends on the refractive index. Due to photoelastic and thermo-optic effects, there is a spectral shift when mechanical stress or temperature changes are applied in the fibre. This provides a useful self-referencing sensor in the frequency domain, but has the drawback that the spectral shift caused by strain or temperature has the same sign. So, it is difficult to isolate strain or temperature effects using a single FBG sensor.

An adequate measurement of both temperature and strain requires a suitable sensor with differential sensitivity for each parameter. Several designs have been proposed for the simultaneous measurements of two or more parameters, using gratings in fibre optics. Among such designs, we can mention the use of Bragg gratings coupled to rocking filters [4], two Bragg gratings with an in-fibre Fabry-Perot interferometer [5], Bragg gratings written in polymer and silica fibres [6] and Bragg gratings with a differential strain [7, 8]. It has been shown that type-I and type-II Bragg gratings can also be used to obtain differential sensitivity to temperature and strain [9].

FBGs written in high birefringence (HiBi) fibre optics have also been described as high efficiency sensors for the simultaneous measurement of those parameters or two strain components [10-14].

The optical reflection spectrum of FBGs written in $\mathrm{HiBi}$ fibres, with nonpolarized light launched into the fibre, has a two-peak structure corresponding to the two orthogonal polarization modes of the fibre. In these fibres, glass regions with high thermal expansion coefficients induce mechanical stress in the core. Due to the intrinsic stress-induced birefringence, the two orthogonal polarizations of the $\mathrm{LP}_{01}$ mode are split and each have a different effective refractive 
index. These linear polarizations are the slow, $\mathrm{LP}_{01}^{\mathrm{X}}$, and fast, $\mathrm{LP}_{01}^{\mathrm{Y}}$, modes associated with the principal directions of the refractive index profile of the fibre, here named $\mathrm{X}$ and $\mathrm{Y}$, respectively. When under strain, the additional birefringence will be added to the intrinsic one, and this will cause a change in the Bragg wavelength of each polarized reflection band. The shift in the Bragg wavelength depends both on the polarization of the band and on the geometry of the strain. Moreover, while temperature and longitudinal strain cause similar shifts in both bands, transverse strain induces different variations in the corresponding Bragg wavelengths. This way it is possible to distinguish the effects of transverse strain and those of longitudinal strain or temperature.

The most common commercial designs of HiBi fibres are the internal elliptical cladding (IEC), PANDA and bow tie fibres. These designs differ in the transverse profile of the refractive index due to the presence of stress-applying elements inserted in the cladding of the fibre optic. The stress profile causes different responses of the induced birefringence when strain and/or temperature are present, which can be used for the simultaneous measurements of both parameters using fibre Bragg gratings [10-14].

The use of a FBG in the HiBi fibre as a fibre optic sensor requires the characterization of the grating properties in the presence of the parameters to be measured, so that a specific calibration for those properties is obtained, to be used later in the sensing process. Optimizing the HiBi fibre characteristics can lead to better performance of a sensor. It has been observed that the birefringence of HiBi fibre optics (of the same family) with different diameters is also different. The difference is due to the fact that the intrinsic stress profile is not the same. In this paper, we investigate the response of FBGs written in HiBi fibres with different diameters, using both commercial and chemically etched fibres. Changes in the stress profile of HiBi fibres due to reduced diameter by etching can modify the response of a FBG sensor system to strain or temperature. Additionally, the study of FBGs in HiBi fibre under chemical etching can lead to useful information about the intrinsic stress behaviour in such fibres.

\section{Grating recording and optical characterization: normal and reduced diameter fibres}

Bragg gratings were recorded in bow tie and IEC HiBi fibre optics with $125 \mu \mathrm{m}$ and $80 \mu \mathrm{m}$ of cladding diameter, obtained from a few commercial providers (3M, Fibercore, Newport).

The FBGs were written by illuminating the fibre under a phase mask by an Excimer laser at $248 \mathrm{~nm}$ [15]. Two phase masks were used to write the gratings, having periods of $1070 \mathrm{~nm}$ and $1072 \mathrm{~nm}$. The estimated length of the gratings is $10 \mathrm{~mm}$, derived from the laser beam diameter.

As a starting point, the FBGs in bow tie HiBi fibre optics were characterized for the two different cladding diameters available. The reflection spectra of such gratings (see figure 1) show the characteristic two-band structure, corresponding to both $\mathrm{X}$ and $\mathrm{Y}$ polarizations (there are low intensity bands shifted to the 'blue' side of the spectrum, in the case of a smaller diameter fibre, but their origin was not investigated). The main difference between the spectra in those fibres is the

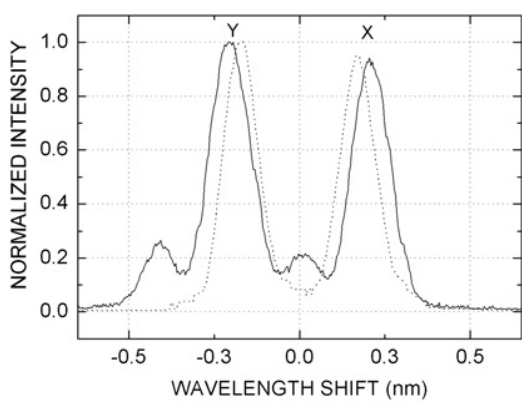

Figure 1. Optical reflection spectra from Bragg gratings in bow tie fibres. Spectra are plotted as a function of the wavelength shift from the central position as the gratings were recorded using different phase masks. Continuous line: $80 \mu \mathrm{m}$ diameter fibre; dashed line: $125 \mu \mathrm{m}$ diameter fibre.

Table 1. Determined birefringence of the fibres used.

\begin{tabular}{ll}
\hline Fibre type (diameter) & Birefringence \\
\hline Bow tie $(125 \mu \mathrm{m})$ & $3.2 \times 10^{-4}$ \\
Bow tie $(80 \mu \mathrm{m})$ & $3.7 \times 10^{-4}$ \\
Bow tie, etched $(81 \mu \mathrm{m})$ & $3.1 \times 10^{-4}$ \\
IEC $(125 \mu \mathrm{m})$ & $5.5 \times 10^{-4}$ \\
IEC $(80 \mu \mathrm{m})$ & $7.1 \times 10^{-4}$ \\
IEC, etched $(82 \mu \mathrm{m})$ & $4.2 \times 10^{-4}$ \\
\hline
\end{tabular}

value of the band separation, which measures the intrinsic stress-induced birefringence.

The birefringence of the fibre was calculated from the Bragg wavelengths for the two polarized reflection bands, using the phase mask period employed during the writing process. Table 1 shows the measured values for that parameter; their difference is almost $16 \%$ (between commercial bow tie fibres of different diameters). It should be noted that the birefringence increases in the fibre with smaller diameter.

The results for IEC fibres are similar (see table 1). However, the birefringence obtained for the $80 \mu \mathrm{m}$ diameter fibre is approximately $29 \%$ greater than that of the standard fibre.

One point to remark is that the observed birefringence for bow tie fibres is relatively smaller than for IEC fibres, and other measurements have also shown that it is smaller than the birefringence of PANDA fibres [11].

In order to investigate the changes in the birefringence properties of $\mathrm{HiBi}$ fibres as a function of the fibre optic diameter, samples of bow tie and IEC fibres were chemically etched in hydrofluoric acid (HF), while the optical spectra of pre-recorded Bragg gratings were measured. The etching was done for two different acid concentrations, $20 \%$ and $40 \%$ (per volume parts). Optical spectra were recorded using a LED as the light source, an optical circulator and a conventional optical spectrum analyser. The diameter of the fibres during the etching was measured by having several pristine samples of the fibre in the acid. Samples were removed successively from the acid, rinsed in distilled water, dried, and then measured under a microscope with a calibrated scale.

An initial test was conducted with a sample of each fibre type, where a FBG was previously written, etched for $15 \mathrm{~min}$ in a solution of $40 \% \mathrm{HF}$. The final diameter of the sample was $81 \mu \mathrm{m}$ for the bow tie fibre, and $82 \mu \mathrm{m}$ for the IEC fibre sample. 


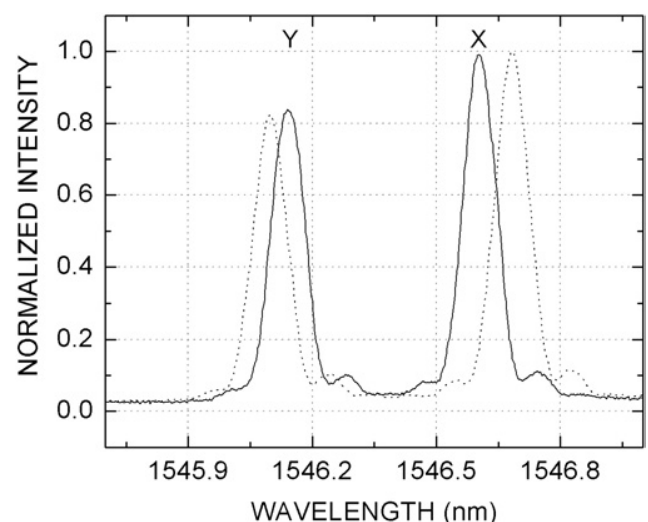

Figure 2. Reflection spectra of a FBG in a reduced diameter IEC fibre ( $82 \mu \mathrm{m}$, continuous line) compared to a normal diameter one (125 $\mu \mathrm{m}$, dashed line). The HiBi fibre was etched in $40 \% \mathrm{HF}$ concentration.

Figure 2 shows a comparison of FBG reflection spectra in the IEC fibre, before and after the chemical etching; the obtained birefringence values for both samples are also included in table 1.

Figure 3 shows the changes in the transverse section of the fibres after approximately the same exposure time to the $20 \%$ $\mathrm{HF}$ concentration etching. It is clear from the photographs that the etching is more pronounced in the IEC fibre, which also shows a higher asymmetry on the borders close to the axes along the major axis of the internal elliptical cladding. The internal cladding was almost exposed, reducing the integrity of the outer cladding (the dark regions on the left of the photograph also suggest accelerated etching to the internal elliptic region). On the other hand, the bow tie sample showed reduced corrosion due to the acid and the smaller stressapplying region was still well confined by the outer cladding.

The result for the chemically etched bow tie fibre was similar to those obtained for the standard diameter fibre; the change in the birefringence due to the etching is negligible (see table 1). This is probably due to the inner position of the stress-applying elements, whose external diameter reaches only $37 \mu \mathrm{m}$; the etching reduced the fibre diameter but the final value was far from those elements, so that no discernible change in the stress distribution was expected.
The striking point for IEC fibres is that the separation between the reflection bands in the optical reflection spectra decreased, that is, the intrinsic birefringence for the thinned fibres is smaller than that of the normal diameter fibre. The latter behaviour, as compared to the commercial, $80 \mu \mathrm{m}$ diameter, fibres used in this work, implies that the difference between the stress components along the major and minor axes of the stress-applying region is reduced as the outer cladding is removed, releasing the intrinsic stress over the fibre core.

Previous studies with IEC fibres have revealed that a strong differential stress $\left(\sigma_{x}-\sigma_{y}\right)$ exists in the interface between the external cladding and the elliptical stress-applying region of IEC fibres, but that behaviour has been observed only along the major axis of the ellipse [16].

For the IEC sample, the largest axis of the ellipse measures around $75 \mu \mathrm{m}$. It can be seen that the ellipse is still discernible even when the etching approaches its border. However, further etching has increased the rate, probably because regions with higher internal stress came in contact with the acid (it is well known that the etching is faster in regions with high stress) and also because of the different composition of the glass in the internal stress-applying region. So it is possible to etch the middle of the fibre faster (along the major axis of the ellipse in figure 3).

If the fibre is not removed from the acid, two fibre structures, joined to the original fibre in a position close to the immersion level, are obtained. The latter result is consistent with other experiments using D-shaped fibres with internal elliptical cladding [17]. The authors of that paper have shown the etching of the fluorine doped internal region, up to its complete removal, even if the outside cladding (pure silica) maintained its integrity.

Figure 4 presents the reduced rate etching for IEC and bow tie fibres. HF acid was diluted to $20 \%$ (parts per volume) in order to reduce the velocity of chemical etching and to increase the sampling points along the process (note the difference in the time scale of both graphs).

\subsection{Internal elliptical cladding fibre}

The spectra in figure 5(a) show the peak wavelength position for each polarization band when the HF exposure time

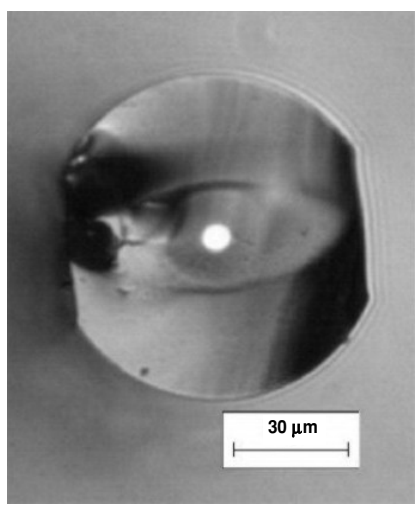

(a)

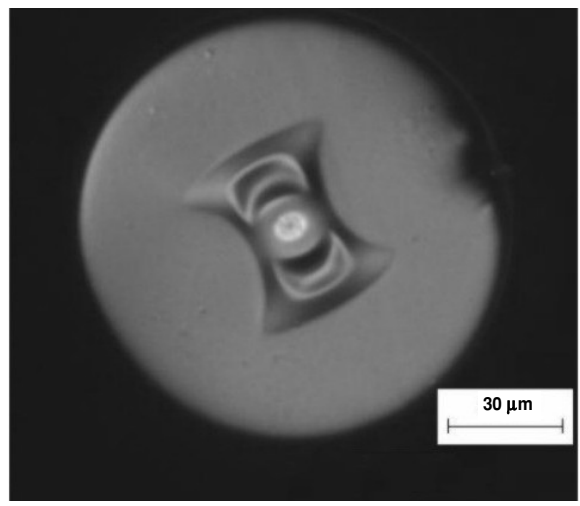

(b)

Figure 3. Microphotographs of the transverse section of etched HiBi fibres: $(a)$ IEC fibre, $86 \mu$ m diameter; $(b)$ bow tie fibre, diameter $110 \mu \mathrm{m}$. 




(a)



(b)

Figure 4. Diameter of HiBi fibres as a function of the exposure time: (a) IEC fibre, $(b)$ bow tie fibre. HF concentration was $20 \%$.

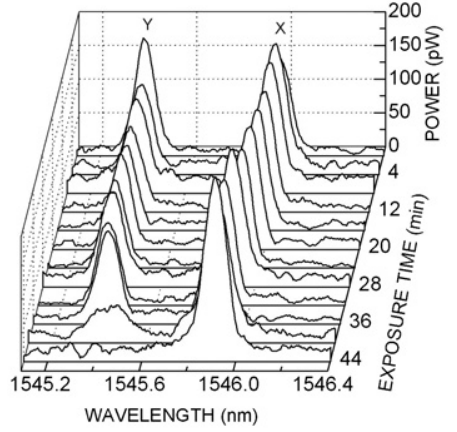

(a)

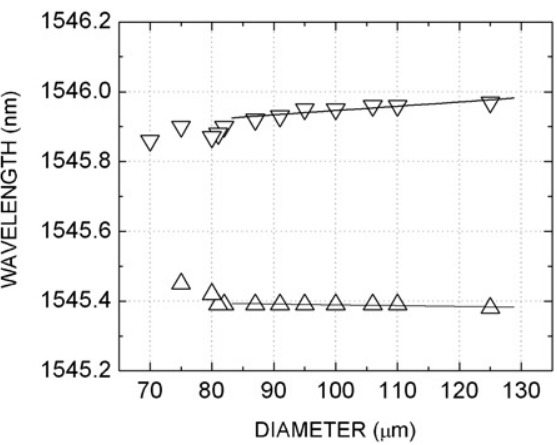

(b)

Figure 5. (a) Evolution of the reflection bands of Bragg gratings written in IEC fibre as a function of the HF etching time. HF concentration was $20 \%$. (b) Peak position of X-polarized $(\nabla)$ and Y-polarized $(\Delta)$ bands of FBGs in the IEC fibre as a function of the fibre diameter, reduced by chemical etching: symbols_experimental points, lines-linear regression.

increases. Initially, a small reduction in the band separation (which corresponds to the fibre birefringence) is perceived. The process accelerates after approximately $10 \mathrm{~min}$. It can also be observed that the intensity of the Y-polarized band (shorter wavelengths) decreases, while the intensity of the other band has a small increase.

After 36 min of exposure, the optical spectrum presents now a single band, which means that the fibre's birefringence is approximately null. It is interesting to note that the remaining band is the one that corresponds to the initial $\mathrm{X}$ polarization, associated with light polarized along the major axis of the ellipse in figure 3(a).

The chemical etching along the direction of the major axis, which reaches the internal region of the fibre faster, releases the stress causing the vanishing of the other band. The changes in the birefringence, measured from the Bragg wavelengths, can be related to the modification of the elliptical structure and therefore with the variation of the internal stress in IEC fibres.

The FBG properties change dramatically after the fibre diameter reaches approximately $75 \mu \mathrm{m}$ (40 min exposure to acid). The band associated with the $\mathrm{Y}$ polarization broadens, its peak intensity almost vanishes while the central wavelength shifts, and the band collapses into the X-polarized band. Figure $5(b)$ shows the changes in the peak position of the reflected polarized bands as a function of the IEC fibre diameter. The first point on the graph shows only a single band, related to the vanishing of the fibre intrinsic birefringence (spectrum in the foreground in figure $5(a)$ ).

In figure $5(b)$, the peak positions of both reflection bands of the FBG are plotted as a function of the fibre diameter during the etching. It can be seen that the change is almost linear when the diameter is greater than $85 \mu \mathrm{m}$. The slopes of the best-fit lines are $1.24 \mathrm{pm} \mu \mathrm{m}^{-1}$ for $\mathrm{X}$ polarization and $-0.23 \mathrm{pm} \mu \mathrm{m}^{-1}$ for the Y-polarized band.

The different slopes for the $\mathrm{X}$ - and Y-polarized bands can be related to asymmetric changes of the internal stress applied by the internal elliptical cladding. The higher (absolute) slope for the X-polarized band implies that initially the reduction is greater along the major axis of the ellipse. However, the situation changes rapidly when the core diameter approaches $80 \mu \mathrm{m}$, and the Y-polarized band disappears. The faster rate agrees with the optical observation of the etched samples, where the attack along the minor axis of the ellipse can lead to the core destruction and separation of the remaining halves.

\subsection{Bow tie fibre}

The effect of chemical etching in the optical spectrum of Bragg gratings written in bow tie fibres is shown in figures $6(a)-(b)$. However, as the etching rate was lower in this experiment, the changes in the spectral bands are perceived with greater detail. It is clear from figure $6(a)$ that the two polarization bands effectively collapse. The peak intensity of the Y-polarized band decreases as its Bragg wavelength moves to the X-polarized band.

Initially both bands show a trend to longer wavelengths on their peak position, as the diameter changes from $100 \mu \mathrm{m}$ to $65 \mu \mathrm{m}$, but the apparent slope is higher for the Y-polarized band (as the reaction temperature was not measured, and the reaction time was far greater in this case, it is possible to have thermal changes affecting the peak position). Further etching causes the X-polarized band to shift sharply to shorter 


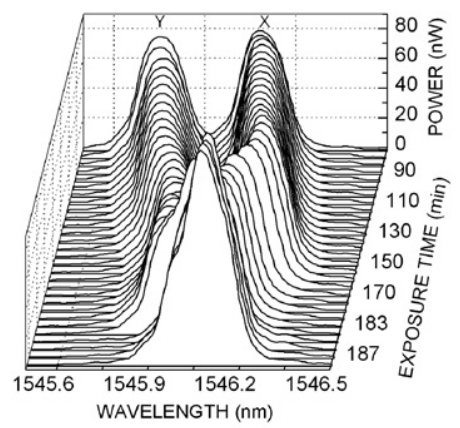

(a)

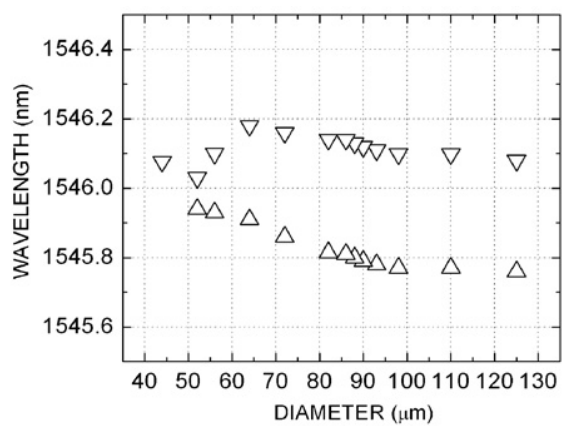

(b)

Figure 6. (a) Evolution of the reflection bands of Bragg gratings, written in a bow tie fibre, as a function of the HF etching time. HF concentration was $20 \%$. (b) Peak position of X-polarized $(\nabla)$ and Y-polarized $(\Delta)$ bands of FBGs in the bow tie fibre as a function of the fibre diameter, reduced by chemical etching: symbols—experimental points.



(a)

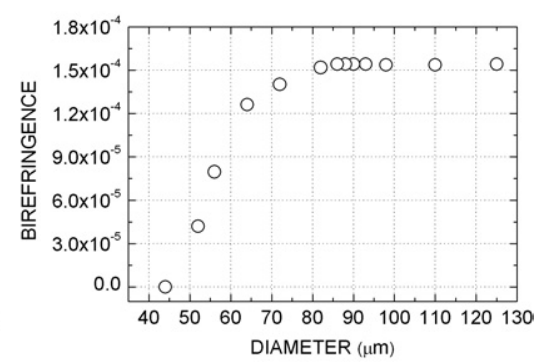

(b)

Figure 7. Measured birefringence of etched HiBi fibres plotted as a function of their diameter: $(a)$ IEC fibre and $(b)$ bow tie fibre. Etching by $\mathrm{HF}$ in concentrations of $20 \%(0)$ and $40 \%(\nabla)$.

wavelengths, until both bands collapse when the diameter reaches approximately $45 \mu \mathrm{m}$ (figure $6(b))$.

The other striking difference from the previous case is that the final peak position of the resulting band is now somewhere in the middle of the original positions of each polarization band, whereas for the IEC fibre it is localized approximately at the original X polarization Bragg wavelength. This means that the etching in the bow tie fibre affects the internal stress profile in a more regular way. The smaller intrinsic stress-applying region is more protected as the outer cladding is removed. Also, it shall be considered that even at the ultimate obtained diameter $(\approx 40 \mu \mathrm{m})$ the cladding maintains its azimuthal integrity (while for the IEC fibre it has been destroyed at the last measured point).

\subsection{Birefringence comparison}

The behaviour described in sections 2.1 and 2.2 agrees with the intrinsic birefringence measurements obtained from FBGs in HiBi fibres [18] and from previous measurements concerning such fibres [16], which show IEC HiBi fibres as having the highest stress-induced birefringence, that is, the difference between stress components along the principal axes of the refractive index profile is also the highest. Chemical etching is also influenced by the stress distribution in the glass. Furthermore, other authors [16] mentioned that, in the cladding region close to the border of the elliptical region, the differential stress causes a tendency to generate cracks when the fibre is cleaved. This fact also supports the evidence for faster cladding etching along the major axis of the internal elliptical cladding region.

Figures $7(a)$ and $(b)$ show the birefringence as a function of the diameter for the etching in $20 \% \mathrm{HF}$ concentration. The results of the two measurements in $40 \%$ concentration are also shown for the IEC fibres. The vanishing of the intrinsic stress-induced birefringence is more evident for the bow tie fibre, due to the reduced reaction rate during the etching.

In agreement with the previous discussion, with diameters between $125 \mu \mathrm{m}$ and $85 \mu \mathrm{m}$, the birefringence for the IEC fibre also decreases almost linearly. Further reduction in the diameter causes a quick reduction in the birefringence that vanishes at diameter values close to $75 \mu \mathrm{m}$. The situation is slightly different for the bow tie fibre that practically maintains its stress-induced birefringence constant until $85 \mu \mathrm{m}$, and then it decreases until it vanishes. However, it is to be noted that both fibres have vanishing birefringence for diameters that are close to the value of the maximum dimension of the stressapplying region, in accordance with the supposition that the outer cladding is an important player in the internal stress profile.

\section{Grating characterization under transverse strain and temperature}

One of the characteristics of the gratings written in HiBi fibres is their asymmetric spectral response to an applied transverse load. Due to the non-homogeneous structure of the fibre, the internal stress variations and, consequently, the deformation 
Table 2. Sensitivities to temperature and strain with etched gratings.

\begin{tabular}{lllll}
\hline & \multicolumn{4}{c}{ Spectral sensitivities } \\
\cline { 2 - 5 } Fibre type (diameter) & $\begin{array}{l}\partial \lambda_{X} / \partial T \\
\left(\mathrm{pm}^{\circ} \mathrm{C}^{-1}\right)\end{array}$ & $\begin{array}{l}\partial \lambda_{Y} / \partial T \\
\left(\mathrm{pm}{ }^{\circ} \mathrm{C}^{-1}\right)\end{array}$ & $\begin{array}{l}\partial \lambda_{X} / \partial \varepsilon \\
\left(\mathrm{pm} \mu \varepsilon^{-1}\right)\end{array}$ & $\begin{array}{l}\partial \lambda_{Y} / \partial \varepsilon \\
\left(\mathrm{pm} \mu \varepsilon^{-1}\right)\end{array}$ \\
\hline Etched IEC $(82 \mu \mathrm{m})$ & 7.00 & 6.90 & -0.70 & 2.23 \\
& & & 3.40 & -0.10 \\
Bow tie $(80 \mu \mathrm{m})$ & 8.02 & \multirow{2}{*}{8.46} & -0.02 & 1.16 \\
& & & 1.20 & 0.30 \\
Standard IEC $(125 \mu \mathrm{m})$ & 6.76 & 6.71 & -0.29 & 1.55 \\
& & & 7.02 & 1.02 \\
\hline
\end{tabular}

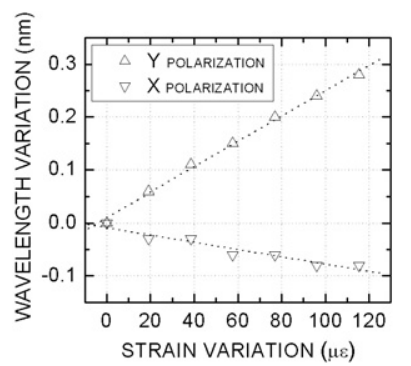

(a)

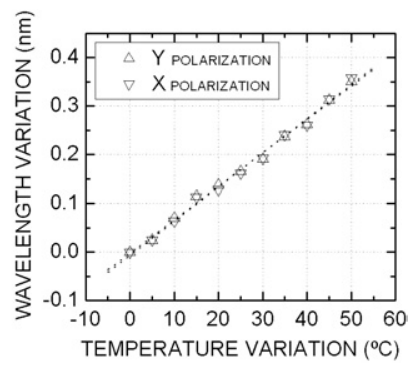

(b)
Figure 8. Dependence of the Bragg wavelengths in the IEC HiBi fibre thinned by chemical etching, as a function of the magnitude of the applied transverse strain $(a)$ and temperature $(b)$. Load direction of application: $X$-axis $(\nabla)$ and $Y$-axis $(\Delta)$.

along each of the main axes of the fibre will be different $[11,18]$. When a load is applied to one of the main axes, $X$ or $Y$, it leads to a variation in one of the bands in the reflection spectrum of the grating, whereas the other band will present smaller changes.

The change in the Bragg wavelength of the reflection spectrum of the FBG, due to a temperature variation $\Delta T$ and strain $\Delta \varepsilon$, for each polarization, is given by

$$
\begin{aligned}
\Delta \lambda_{X} & =\frac{\partial \lambda_{X}}{\partial T} \Delta T+\frac{\partial \lambda_{X}}{\partial \varepsilon} \Delta \varepsilon \\
\Delta \lambda_{Y} & =\frac{\partial \lambda_{Y}}{\partial T} \Delta T+\frac{\partial \lambda_{Y}}{\partial \varepsilon} \Delta \varepsilon
\end{aligned}
$$

where $\partial \lambda_{X} / \partial T$ and $\partial \lambda_{Y} / \partial T$ are the temperature variation coefficients and $\partial \lambda_{X} / \partial \varepsilon$ and $\partial \lambda_{Y} / \partial \varepsilon$ are the deformation coefficients.

The transverse load used to characterize a FBG in reduced diameter HiBi fibres is performed by a micro-scratch mechanical system. The system uses an arm to apply a load to the sample with a precision of $0.1 \mathrm{~N}$. The grating $(82 \mu \mathrm{m}$ diameter fibre) lies between two plates having a length of $13 \mathrm{~mm}$. Two other fibres are placed parallel to the FBG to serve as a guide and to avoid tilt in the upper plate, while the apparatus arm applies the load to it.

The measurements are made for the fibre oriented with the fast or slow birefringence axis in the direction of the applied load.

Peak positions of the reflection spectrum for $\mathrm{X}$ and $\mathrm{Y}$ polarization bands are shown in figure $8(a)$, for the two directions of the applied load. Such measurements are done at a constant temperature.

The temperature dependence of the reflection bands of the FBG is also characterized using a cooling/heating system.
That system is composed of two thermoelectric coolers, controlled by a dedicated electronic circuit. Calibration lines for the temperature-induced shifts in the Bragg wavelengths are shown in figure $8(b)$. The corresponding slopes (sensitivity of the Bragg wavelength to temperature changes) are almost the same as determined for gratings in normal diameter fibres.

The slopes of calibration lines for strain and temperature are given in table 2, where the slopes of calibration lines for strain and temperature, obtained with other types of HiBi fibres (commercial samples), are also shown for comparison [11]. The opposite signal values, as compared to the pictured slopes in figure 8 , are due to the definition of the strain axes. It can be seen that the slopes of strain calibration lines for the load applied along the $X$-axis of reduced diameter fibres are higher than the corresponding slopes in the standard HiBi FBG sensors [11], while for the load applied along the $Y$-axis, the opposite behaviour occurs.

The fact that the FBG sensitivity to temperature is the same for both polarized bands, whereas sensitivity to strain depends both on the band polarization and on the direction of the applied load, allows the FBG in the HiBi fibre to measure simultaneously transverse strain and temperature. Moreover, depending on the orientation, sensitivity can be relatively higher to normal diameter fibres.

\section{Simultaneous measurement of transverse strain and temperature using an etched HiBi fibre}

The FBG in an IEC HiBi fibre thinned by chemical etching was applied as a sensor to simultaneously measure the transverse strain and temperature.

Equations (1) can be rearranged and written in matrix form, in order to calculate the transverse strain and temperature, given the measured wavelength shifts for each polarization band:

$$
\left[\begin{array}{c}
\Delta T \\
\Delta \varepsilon
\end{array}\right]=\mathbf{K}^{-1}\left[\begin{array}{c}
\Delta \lambda_{X} \\
\Delta \lambda_{Y}
\end{array}\right]
$$

where the non-singular matrix of the FBG spectral sensitivities, $\mathbf{K}$, is given by

$$
\mathbf{K}=\left[\begin{array}{ll}
\frac{\partial \lambda_{X}}{\partial T} & \frac{\partial \lambda_{X}}{\partial \varepsilon} \\
\frac{\partial \lambda_{Y}}{\partial T} & \frac{\partial \lambda_{Y}}{\partial \varepsilon}
\end{array}\right] .
$$

The system performance is evaluated when the sensing FBG is simultaneously subjected to strain and temperature changes. Gratings previously characterized were subject to the microscratch apparatus. While the transverse load is applied over 
Bragg gratings in normal and reduced diameter high birefringence fibre optics

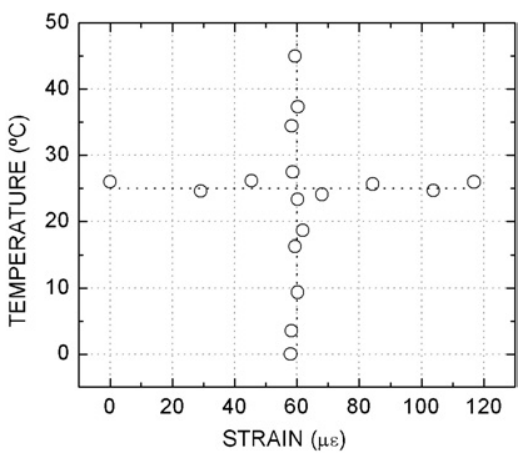

Figure 9. Sensor output as determined by equation (1) for the applied strain at constant temperature and temperature changing at constant strain.

Table 3. Simultaneous measurements of temperature and strain using a grating in etched $\mathrm{HiBi}$ fibre. Figures shown in the border cells are applied by the equipment. Inner cells present the respective measurements.

\begin{tabular}{lccccc}
\hline Set values & $16^{\circ} \mathrm{C}$ & $26^{\circ} \mathrm{C}$ & $36^{\circ} \mathrm{C}$ & $46^{\circ} \mathrm{C}$ & $56^{\circ} \mathrm{C}$ \\
\hline $33 \mu \varepsilon$ & $15^{\circ} \mathrm{C}$ & $28^{\circ} \mathrm{C}$ & $33^{\circ} \mathrm{C}$ & $44^{\circ} \mathrm{C}$ & $56^{\circ} \mathrm{C}$ \\
& $37 \mu \varepsilon$ & $37 \mu \varepsilon$ & $39 \mu \varepsilon$ & $42 \mu \varepsilon$ & $42 \mu \varepsilon$ \\
$48 \mu \varepsilon$ & $17^{\circ} \mathrm{C}$ & $29^{\circ} \mathrm{C}$ & $36^{\circ} \mathrm{C}$ & $47^{\circ} \mathrm{C}$ & $56^{\circ} \mathrm{C}$ \\
& $54 \mu \varepsilon$ & $57 \mu \varepsilon$ & $56 \mu \varepsilon$ & $54 \mu \varepsilon$ & $59 \mu \varepsilon$ \\
$64 \mu \varepsilon$ & $17^{\circ} \mathrm{C}$ & $28^{\circ} \mathrm{C}$ & $36^{\circ} \mathrm{C}$ & $46^{\circ} \mathrm{C}$ & $57^{\circ} \mathrm{C}$ \\
& $49 \mu \varepsilon$ & $48 \mu \varepsilon$ & $48 \mu \varepsilon$ & $54 \mu \varepsilon$ & $53 \mu \varepsilon$ \\
$79 \mu \varepsilon$ & $17^{\circ} \mathrm{C}$ & $29{ }^{\circ} \mathrm{C}$ & $36^{\circ} \mathrm{C}$ & $48^{\circ} \mathrm{C}$ & $58^{\circ} \mathrm{C}$ \\
& $66 \mu \varepsilon$ & $80 \mu \varepsilon$ & $71 \mu \varepsilon$ & $65 \mu \varepsilon$ & $73 \mu \varepsilon$ \\
$94 \mu \varepsilon$ & $17^{\circ} \mathrm{C}$ & $29{ }^{\circ} \mathrm{C}$ & $36^{\circ} \mathrm{C}$ & $48^{\circ} \mathrm{C}$ & $58^{\circ} \mathrm{C}$ \\
& $80 \mu \varepsilon$ & $100 \mu \varepsilon$ & $94 \mu \varepsilon$ & $91 \mu \varepsilon$ & $85 \mu \varepsilon$ \\
\hline
\end{tabular}

the fibre, the cooling/heating system was placed outside the holding plate of the fibres in order to avoid any influence from the applied strain. This allows the measurement of the temperature sensitivity of each reflection band, while the strain sensitivity is also measured.

The resulting values of $\Delta \lambda_{X}$ and $\Delta \lambda_{Y}$ were used in equation (2) to obtain the sensor output. For an applied strain, $\Delta \varepsilon$, of $60 \mu \varepsilon$, the temperature was varied in a $50{ }^{\circ} \mathrm{C}$ range. An analogous procedure was followed with a constant temperature, $T=25{ }^{\circ} \mathrm{C}$, and varying the strain in a $120 \mu \varepsilon$ range. The results are shown in figure 9. The standard deviations were found to be $\pm 0.84{ }^{\circ} \mathrm{C}$ and $\pm 1.26 \mu \varepsilon$ for temperature and strain measurements, respectively.

Table 3 shows the results of simultaneous transverse strain and temperature measurements obtained with matrix $\mathbf{K}$ and the values of $\Delta \lambda_{X}$ and $\Delta \lambda_{Y}$ of the reflection spectra. Each pair of measured strain and temperature can be compared to the preset values whose readings are displayed by a digital thermometer and the micro-scratch system and shown in the border cells.

The strain values have been evaluated with an error of $\pm 16 \mu \varepsilon$ for set values in the interval between 33 and $94 \mu \varepsilon$, while the temperature error has been estimated to be $\pm 3{ }^{\circ} \mathrm{C}$ for set values ranging from 16 to $56{ }^{\circ} \mathrm{C}$. These values can be compared to other results obtained with a normal diameter IEC sensor that has strain values evaluated with an error of $\pm 15 \mu \varepsilon$ for set values in the interval between 61 and $91 \mu \varepsilon$, and the temperature error estimated to be $\pm 4{ }^{\circ} \mathrm{C}$ for set values ranging from 12 to $46{ }^{\circ} \mathrm{C}$ [11]. We believe that the increase in the dynamical range is a result of the reduction of the mechanical resistance of the fibre after thinning.

Whereas the errors using FBGs in normal and reduced diameter HiBi fibres as a sensor are of comparable magnitude, the dynamic range for strain measurements with the latter is almost doubled as compared to the former sensors. This fact is important for technological applications where FBGs can be tailored to attend a specific measurement range.

\section{Conclusion}

FBGs written in a $\mathrm{HiBi}$ fibre have been produced and characterized using IEC and bow tie fibres, with 125 and $80 \mu \mathrm{m}$ external diameter. Changes in the internal stress distribution in these fibres were analysed through the evolution of their reflection spectra. The birefringence of the fibres was obtained as a function of the fibre diameter, during chemical etching. FBGs written in the reduced diameter HiBi fibre were characterized in terms of their response to changes in temperature and transverse strain and, in the latter case, as a function of the direction of load application. The slopes of temperature and strain calibration lines were also determined. Simultaneous measurements of transverse strain and temperature were performed using a FBG written in the reduced diameter HiBi IEC fibre, showing that it can be used as a substitute for $125 \mu \mathrm{m}$ diameter sensors, with an additional advantage of having a higher dynamic range.

\section{Acknowledgments}

The authors acknowledge funding received from FCT (Portugal), CNPq (Brazil) and from the CAPES/GRICES agreement, project 58/00.

\section{References}

[1] Higuera J M L 2002 Handbook of Optical Fibre Sensing Technology (New York: Wiley)

[2] Hill K O and Meltz G 1997 Fiber Bragg grating technology fundamentals and overview J. Lightwave Technol. 15 1263-76

[3] Kashyap R 1999 Fibre Bragg Grating (San Diego, CA: Academic)

[4] Kanellopoulos S E, Handerek V A and Rogers A J 1995 Simultaneous strain and temperature sensing with photogenerated in-fibre gratings Opt. Lett. 20 333-5

[5] Du W C, Tao X M and Tau H Y 1999 Fiber Bragg grating cavity sensor for simultaneous measurement of strain and temperature IEEE Photonics Technol. Lett. 11 105-7

[6] Liu H B, Liu H L, Peng G D and Chu P L 2003 Strain and temperature sensor using a combination of polymer and silica fiber Bragg gratings Opt. Commun. 219 139-42

[7] Lo Y L 1998 Using in-fibre Bragg-grating sensors for measuring axial strain and temperature simultaneously on surfaces of structures Opt. Eng. 37 2272-6

[8] Dong X Y, Liu Y, Liu Z and Dong X 2001 Simultaneous displacement and temperature measurement with cantileverbased fiber Bragg grating sensor Opt. Commun. 192 213-7

[9] Frazão O, Lima M J L and Santos J L 2003 Simultaneous measurement of strain and temperature using type I and type IIA fibre Bragg gratings J. Opt. A: Pure Appl. Opt. $5183-5$

[10] Lawrence C M, Nelson D V and Udd E 1997 Measurements of transverse strain with fibre Bragg gratings Proc. SPIE 3042 218-28 
[11] Abe I, Kalinowski H J, Nogueira R, Pinto J L and Frazão O 2003 Production and characterisation of Bragg gratings written in high birefringence fibre optics IEE Proc., Circuits Devices Syst. 12 495-500

[12] Chen G, Liu L, Jia H, Yu J, Xu L and Wang W 2004 Simultaneous strain and temperature measurements with fiber Bragg grating written in novel Hi-Bi optical fiber IEEE Photonics Technol. Lett. 16 221-3

[13] Zhang L, Liu Y, Everall L, Williams J A R and Bennion I 1999 Design and realization of long-period grating devices in conventional and high birefringence fibers and their novel applications as fiber-optic load sensors IEEE J. Sel. Top. Ouantum Electron. 5 1373-8

[14] Chehura E, Ye C C, Staines E S, James S W and Tatam R P 2004 Characterization of the response of fibre Bragg gratings fabricated in stress and geometrically induced high birefringence fibres to temperature and transverse load Smart Mater. Struct. 13 888-95

[15] Araújo F M M 1999 Redes de Bragg em fibra óptica PhD Thesis Department of Physics, University of Porto, Porto, Portugal

[16] Loch M and Heinlein W E 1989 High-resolution measurement of birefringence profiles in stress-induced polarization-maintaining fibres J. Lightwave Technol. 7 1213-6

[17] Smith K H, Selfridge R H, Schultz S M, Markos D J and Ipson B L 2004 Analysis of replacing an optical fiber core with polymer Opt. Express 12 354-60

[18] Abe I, Nogueira R, Pinto J L, Frazão O, Santos J L and Kalinowski H J 2002 Análise espectral de FBGs escritas em fibras HiBi Anais X Simp. Brasileiro de Microondas e Optoeletrônica (Recife, Brazil) pp 75-8 